\title{
Erratum to: Blood or spores? A cautionary note on interpreting cellular debris on human skeletal remains
}

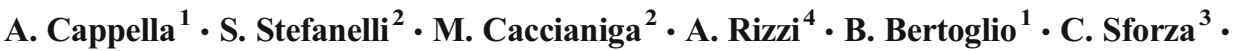 \\ C. Cattaneo ${ }^{1}$
}

Published online: 17 March 2016

(C) Springer-Verlag Berlin Heidelberg 2016

Erratum to: Int J Legal Med (2015) 129:919-926

DOI 10.1007/s00414-014-1140-8

The original version of this article contained errors.

Graph 1 and Figures 8 and 9 captions and their corresponding images did not match. The correct images are now given in this article.

The online version of the original article can be found at $\mathrm{http}$ ://dx.doi.org/ 10.1007/s00414-014-1140-8.

A. Cappella

annalisa.cappella@gmail.com

LABANOF, Laboratorio di Antropologia e Odontologia Forense, Sezione di Medicina Legale e delle Assicurazioni, Dipartimento di Scienze Biomediche per la Salute, Università degli Studi di Milano, Via L. Mangiagalli 37, 20133 Milan, Italy

2 Dipartimento di Bioscienze, Università degli Studi di Milano, Via Celoria 26, Milan, Italy

3 Dipartimento di Scienze Biomediche per la Salute, Università degli Studi di Milano, Via L. Mangiagalli 33, Milan, Italy

4 Dipartimento di Scienze della Terra, CNR (Centro Nazionale Ricerca), Università degli Studi di Milano, Via L. Mangiagalli 31, Milan, Italy 
1. Upper figure on page 924

Fig. 8 One erythrocytewiththe scale bar of $5.00 \mu \mathrm{m}$ (a) and one spore of Dryopteris filix-mas with a 20.00- $\mu \mathrm{m}$ scale bar (b)
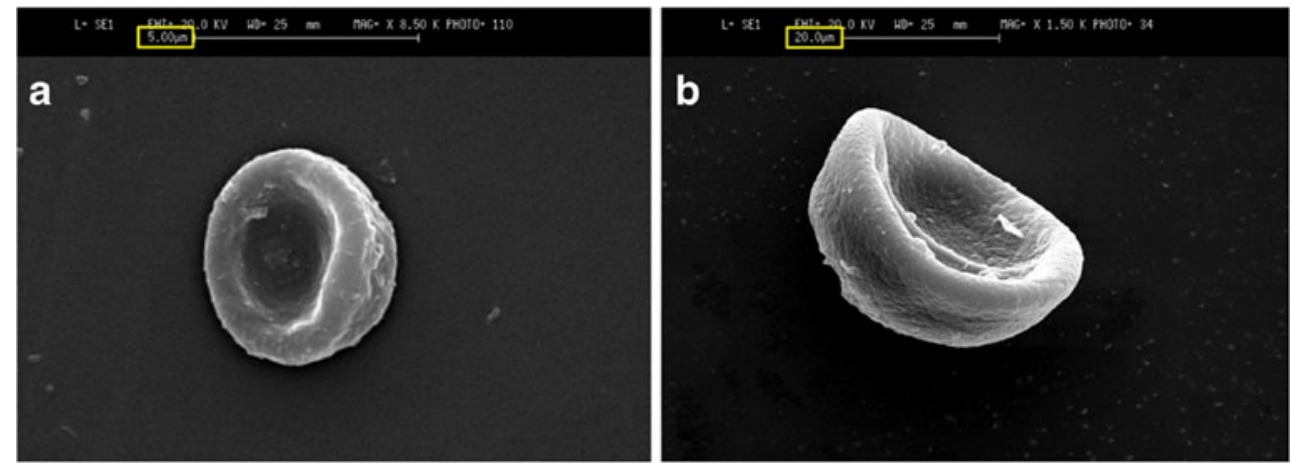

\section{Bottom figure on page 924}

Fig. 9 Chain setting for fungal spores (a) and rouleaux settings for erythrocytes (b)
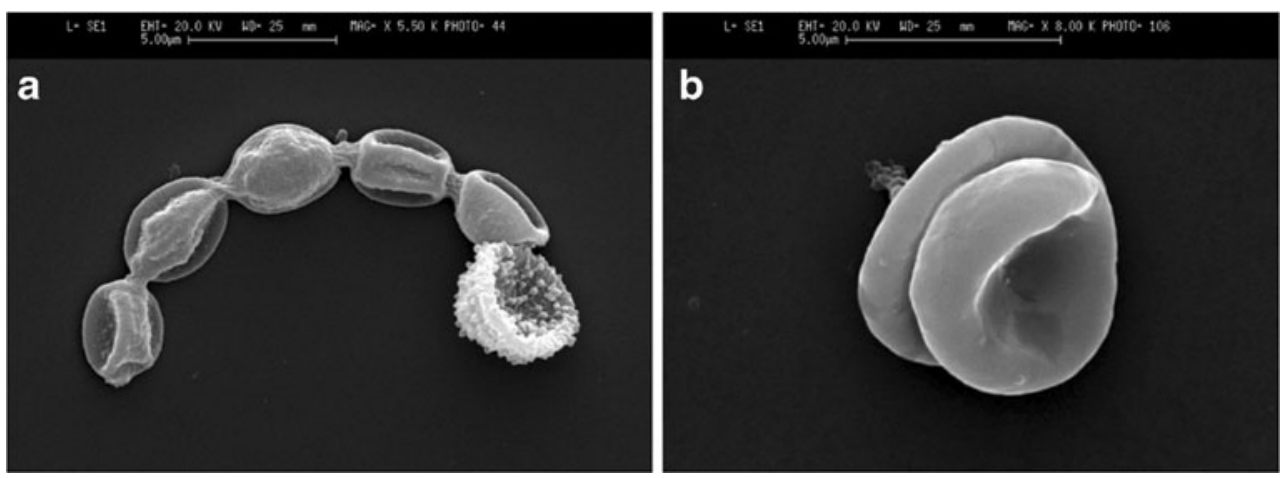

3. Graph on page 925

a

PATHOLOGISTS

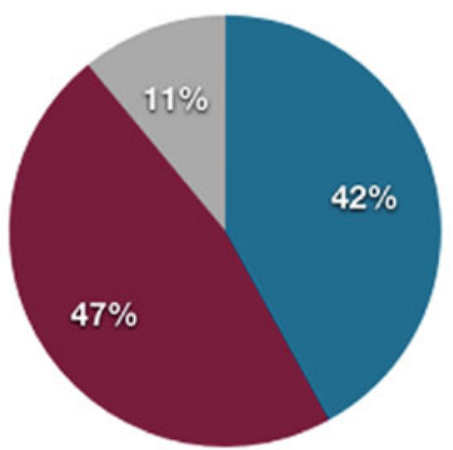

correct

incorrect

b

NATURAL SCIENTISTS

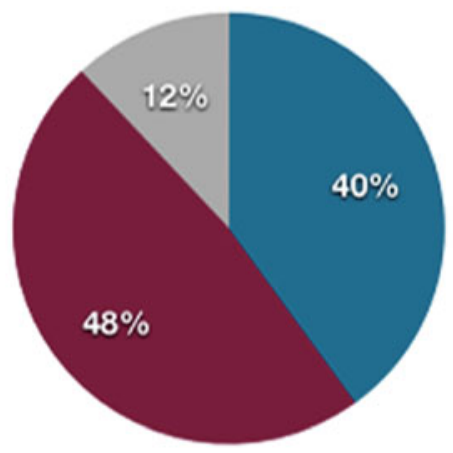

correct

incorrect

uncertain

Graph 1 Percentages of answers for pathologists (a) and natural scientists (b) 